\title{
Effects of rotation and chemical reaction on MHD flow past an inclined plate with variable wall temperature and mass diffusion
}

\author{
U. S. Rajput, Gaurav Kumar * \\ Department of Mathematics and Astronomy, University of Lucknow, U.P, India \\ * Corresponding author: rajputgauravlko@gmail.com
}

\section{Article history}

Received 22 September 2016

Accepted 29 January 2017

\section{Graphical abstract}

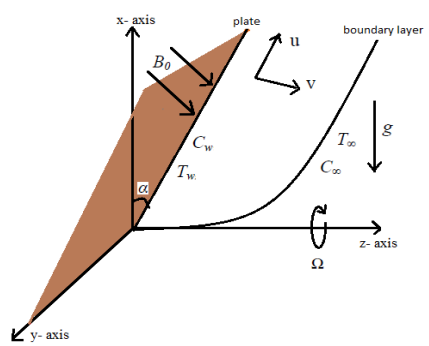

\begin{abstract}
Effects of rotation and chemical reaction on unsteady MHD flow past an impulsively started inclined plate with variable wall temperature and mass diffusion in the presence of Hall current is studied here. Earlier we [7] have studied radiation effects on unsteady MHD flow through porous medium past an oscillating inclined plate with variable temperature and mass diffusion in the presence of Hall current. We obtained the results which were in agreement with the desired flow phenomenon. To study further we are changing the model by considering rotation and chemical reaction. The governing equations involved in the flow model are solved by the Laplace-transform technique. The results obtained have been analyzed with the help of graphs drawn for different parameters. The numerical values obtained for the drag at boundary and Sherwood number have been tabulated. Here too, the results are found to be in agreement with the actual flow.
\end{abstract}

Keywords: MHD flow, rotation, chemical reaction, Hall current.

(C) 2017 Penerbit UTM Press. All rights reserved

\section{INTRODUCTION}

The MHD flow problems play important role in different areas of science and technology. These have many applications in industry, for instance, magnetic material processing, glass manufacturing control processes and purification of crude oil. Hall Effect on free and forced convective flow in a rotating channel was explained by Rao et al. [3] Muthucumarswamy along with Ganesan [4] have studied first order chemical reaction on flow past an impulsively started vertical plate with uniform heat and mass flux. Effect of chemical reaction and heat generation or absorption on double-diffusive convection from a vertical truncated cone in porous media with variable viscosity was analyzed by Mahdy [1]. Effects of chemical reaction, heat and mass transfer and radiation on the MHD flow along a vertical porous wall in the presence of induced magnetic field was developed by Sahin and Chamkha [2]. Zulkhibri et al. [8] have worked on MHD double diffusion flow by free convection past an infinite inclined plate with ramped wall temperature in a porous medium. Hall Effect on unsteady MHD free convection flow over a stretching sheet with variable viscosity and viscous dissipation was presented by Srinivas and Naikoti [5]. Rajput and Kanaujia [6] have examined MHD flow past a vertical plate with variable temperature and mass diffusion in the presence of Hall current. Radiation effect on unsteady MHD flow through porous medium past an oscillating inclined plate with variable temperature and mass diffusion in the presence of Hall current was studied by us [7]. The present study is carried out to examine the effects of rotation and chemical reaction on unsteady MHD flow past an impulsively started inclined plate with variable wall temperature and mass diffusion in the presence of Hall current. The problem is solved by the Laplace transform technique. A selected set of graphical results illustrating the effects of various parameters involved in the problem are presented and discussed. The numerical values of skin-friction and Sherwood number have been tabulated.

\section{MATHEMATICAL ANALYSIS}

The geometrical model of the problem is shown in Figure 1.

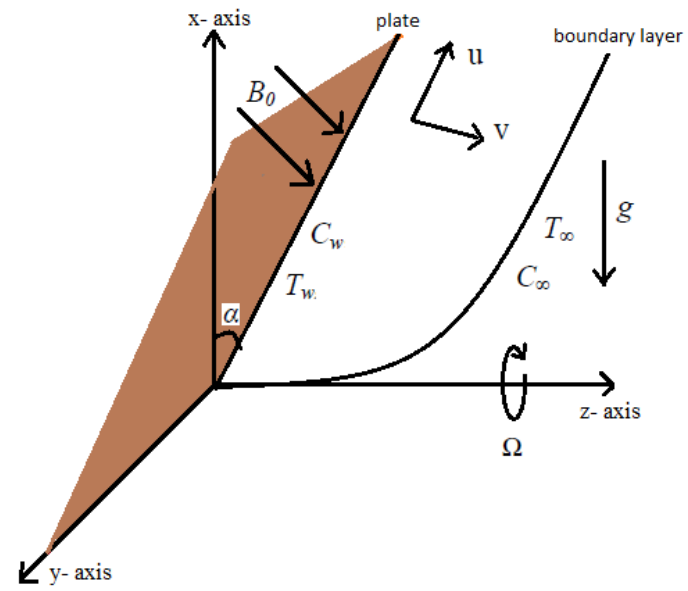

Figure 1 Physical model.

Consider an unsteady flow of a viscous, incompressible, electrically conducting fluid past an impulsively started non-conducting inclined flat plate. The $\mathrm{x}$ axis is taken along the vertical plane and $\mathrm{z}$ axis is normal to it. Thus the $\mathrm{z}$ axis lies in the horizontal plane. The plate is inclined at angle $\alpha$ from vertical. The fluid and the plate rotate as a rigid body with a uniform angular velocity $\Omega$ about z- axis. The magnetic 
field $B_{0}$ of uniform strength is applied perpendicular to the flow. Since the fluid is electrically conducting whose magnetic Reynolds number is very small, therefore the induced magnetic field produced by the fluid motion is negligible in comparison to the applied one. Initially it has been considered that the plate as well as the fluid is at the same temperature $T_{\infty}$. The species concentration in the fluid is taken as $C_{\infty}$. At time $t>0$, the plate starts moving with a velocity $\mathrm{u}_{0}$ in its own plane, and temperature of the plate is raised to $T_{w}$. The concentration $C_{w}$ near the plate is raised linearly with respect to time. The flow model is as under:

$$
\begin{aligned}
& \frac{\partial u}{\partial t}-2 \Omega v=v \frac{\partial^{2} u}{\partial z^{2}}+g \beta \operatorname{Cos} \alpha\left(T-T_{\infty}\right) \\
& +g \beta^{*} \operatorname{Cos} \alpha\left(C-C_{\infty}\right)-\frac{\sigma B_{0}^{2}(u+m v)}{\rho\left(1+m^{2}\right)} \\
& \frac{\partial v}{\partial t}+2 \Omega u=v \frac{\partial^{2} v}{\partial z^{2}}+\frac{\sigma B_{0}^{2}(m u-v)}{\rho\left(1+m^{2}\right)} \\
& \frac{\partial C}{\partial t}=D \frac{\partial^{2} C}{\partial z^{2}}-K_{c}\left(C-C_{\infty}\right) \\
& \rho C_{p} \frac{\partial T}{\partial t}=k \frac{\partial^{2} T}{\partial z^{2}} .
\end{aligned}
$$

The initial and boundary conditions are

$$
\begin{aligned}
& t \leq 0: u=v=0, T=T_{\infty}, C=C_{\infty}, \text { for every z } \\
& t>0: u=u_{0}, v=0 \text {, } \\
& T=T_{\infty}+\left(T_{w}-T_{\infty}\right) A_{0}, \quad \text { at } \quad \mathrm{z}=0 \\
& C=C_{\infty}+\left(C_{w}-C_{\infty}\right) A_{0} \text {, } \\
& u \rightarrow 0, v \rightarrow 0, T \rightarrow T_{\infty}, C \rightarrow C_{\infty} \text { as } z \rightarrow 0
\end{aligned}
$$

Here $u$ and $v$ are the primary and secondary velocities along $\mathrm{x}$ and $\mathrm{z}$ directions respectively, $D$-the mass diffusion coefficient, $K_{c}$ chemical reaction, $C_{w}$-species concentration at the plate $z=0, B_{0}$ - the uniform magnetic field, $\sigma$ - electrical conductivity $C$ - species concentration in the fluid, $\nu$ - the kinematic viscosity, $\rho$ - the density, $C_{p}$ - the specific heat at constant pressure, $g$-the acceleration due to gravity, $\beta$-volumetric coefficient of thermal expansion, $t$-time, $\mathrm{m}$-the Hall current parameter, $T_{w}$ - temperature of the plate at $z=0, T$-temperature of the fluid, $\beta^{*}$ volumetric coefficient of concentration expansion, $k$ - thermal conductivity of the fluid.

The following non-dimensional quantities are introduced to transform equations (1), (2), (3) and (8) into dimensionless form:

$$
\left.\begin{array}{l}
\bar{z}=\frac{z u_{0}}{v}, \bar{u}=\frac{u}{u_{0}}, \bar{v}=\frac{v}{u_{0}}, \theta=\frac{\left(T-T_{\infty}\right)}{\left(T_{w}-T_{\infty}\right)}, S_{c}=\frac{v}{D}, \\
\mu=\rho v, P_{r}=\frac{\mu c_{p}}{k}, G_{r}=\frac{g \beta v\left(T_{w}-T_{\infty}\right)}{u_{0}{ }^{3}}, M=\frac{\sigma B_{0}^{2} v}{\rho u_{0}^{2}}, \\
G_{m}=\frac{g \beta^{*} v\left(C_{w}-C_{\infty}\right)}{u_{0}^{3}}, \bar{C}=\frac{\left(C-C_{\infty}\right)}{\left(C_{w}-C_{\infty}\right)}, K_{0}=\frac{v K_{c}}{u_{0}^{2}}, \\
\bar{t}=\frac{t u_{0}^{2}}{v}, \bar{\Omega}=\frac{v \Omega}{u_{0}^{2}} .
\end{array}\right\}
$$

The symbols in dimensionless form are as under:

$\bar{u}$ - primary velocity, $\bar{v}$ - secondary velocity, $\Omega$ - rotation parameter,

$P_{r^{-}}$Prandtl number, $S_{c^{-}}$Schmidt number, $R$ - Radiation parameter, $\overline{\boldsymbol{t}}$ time, $\theta$ - temperature, $\bar{C}$ - concentration, $G_{r}$-thermal Grashof number,
$G_{m^{-}}$mass Grashof number, $\mu$ - coefficient of viscosity, $M$ - magnetic parameter.

The flow model in dimensionless form is

$$
\begin{aligned}
& \frac{\partial \bar{u}}{\partial \bar{t}}-2 \bar{\Omega} \bar{v}=\frac{\partial^{2} \bar{u}}{\partial \bar{z}^{2}}+G_{r} \operatorname{Cos} \alpha \theta+G_{m} \operatorname{Cos} \alpha \bar{C}-\frac{M(\bar{u}+m \bar{v})}{\left(1+m^{2}\right)} \\
& \frac{\partial \bar{v}}{\partial \bar{t}}+2 \bar{\Omega} \bar{u}=\frac{\partial^{2} \bar{u}}{\partial \bar{z}^{2}}+\frac{M(m \bar{u}-\bar{v})}{\left(1+m^{2}\right)} \\
& \frac{\partial \bar{C}}{\partial \bar{t}}=\frac{1}{S_{c}} \frac{\partial^{2} \bar{C}}{\partial \bar{z}^{2}}-K_{0} \bar{C} \\
& \frac{\partial \theta}{\partial \bar{t}}=\frac{1}{P_{r}} \frac{\partial^{2} \theta}{\partial \bar{z}^{2}}
\end{aligned}
$$

The corresponding boundary conditions (5) become:

$$
\left.\begin{array}{l}
\bar{t} \leq 0: \bar{u}=\bar{v}=0, \theta=0, \bar{C}=0, \text { for every } \bar{z}, \\
\bar{t}>0: \bar{u}=1, \bar{v}=0, \theta=\bar{t}, \bar{C}=\bar{t}, \text { at } \bar{z}=0, \\
\bar{u} \rightarrow 0, \bar{v} \rightarrow 0, \theta \rightarrow 0, \bar{C} \rightarrow 0 \text { as } \bar{z} \rightarrow \infty
\end{array}\right\}
$$

Dropping bars in the above equations, we get

$$
\begin{aligned}
& \frac{\partial u}{\partial t}-2 \Omega v=\frac{\partial^{2} u}{\partial z^{2}}+G_{r} \operatorname{Cos} \alpha \theta+G_{m} \operatorname{Cos} \alpha C-\frac{M(u+m v)}{\left(1+m^{2}\right)} \\
& \frac{\partial v}{\partial t}+2 \Omega u=\frac{\partial^{2} v}{\partial z^{2}}+\frac{M(m u-v)}{\left(1+m^{2}\right)} \\
& \frac{\partial C}{\partial t}=\frac{1}{S_{c}} \frac{\partial^{2} C}{\partial z^{2}}-K_{0} C \\
& \frac{\partial \theta}{\partial t}=\frac{1}{P_{r}} \frac{\partial^{2} \theta}{\partial z^{2}}
\end{aligned}
$$

The boundary conditions are

$\left.\begin{array}{l}t \leq 0: u=v=0, \theta=0, C=0, \text { for every z, } \\ t>0: u=1, v=0, \theta=t, C=t, \text { at } \mathrm{z}=0, \\ u \rightarrow 0, v \rightarrow 0, \theta \rightarrow 0, C \rightarrow 0, \text { as } z \rightarrow \infty\end{array}\right\}$

Writing the equations (12) and (13) in combined form (using $q=u+i v$ )

$$
\begin{aligned}
& \frac{\partial q}{\partial t}=\frac{\partial^{2} q}{\partial z^{2}}+G_{r} \operatorname{Cos} \alpha \theta+G_{m} \operatorname{Cos} \alpha C-q\left(\frac{M(1-i m)}{1+m^{2}}+2 i \Omega\right) \\
& \frac{\partial C}{\partial t}=\frac{1}{S_{c}} \frac{\partial^{2} C}{\partial z^{2}}-K_{0} C \\
& \frac{\partial \theta}{\partial t}=\frac{1}{P_{r}} \frac{\partial^{2} \theta}{\partial z^{2}}
\end{aligned}
$$

The boundary conditions become:

$\left.\begin{array}{l}t \leq 0: q=0, \theta=0, C=0, \text { for every } \mathrm{z}, \\ t>0: q=1, \theta=t, C=t, \quad \text { at } \mathrm{z}=0,\end{array}\right\}$ 
$q \rightarrow 0, \theta \rightarrow 0, C \rightarrow 0$, as $z \rightarrow \infty$

The dimensionless governing equations (17) to (19), subject to the boundary conditions (20), are solved by the usual Laplace transform technique. The solution obtained is as under:

$$
\begin{aligned}
\theta= & t\left\{\left(1+\frac{z^{2} P_{r}}{2 t}\right) \operatorname{erfc}\left[\frac{\sqrt{P_{r}}}{2 \sqrt{t}}\right]-\frac{z \sqrt{P_{r}}}{\sqrt{\pi \sqrt{t}}} e^{-\frac{z^{2}}{4 t}} P_{r}\right\} \\
C= & \frac{e^{-z \sqrt{S_{c} K_{0}}}}{4 \sqrt{K_{0}}}\left\{\operatorname{erfc}\left[\frac{z \sqrt{S_{c}}-2 t \sqrt{K_{0}}}{2 \sqrt{t}}\right]\left(-z \sqrt{S_{c}}+2 t \sqrt{K_{0}}\right)\right. \\
& \left.+e^{2 z \sqrt{S_{c} K_{0}}} \operatorname{erfc}\left[\frac{z \sqrt{S_{c}}+2 t \sqrt{K_{0}}}{2 \sqrt{t}}\right]\left(z \sqrt{S_{c}}+2 t \sqrt{K_{0}}\right)\right\} \\
q= & \frac{e^{-\sqrt{a z}} A_{15}}{4}+\frac{G_{r} \operatorname{Cos} \alpha}{4 a^{2}}\left[z A_{11}+2 e^{-\sqrt{a} z} A_{2} P_{r}+2 A_{14} A_{4}\right. \\
& \left.\left(1-P_{r}\right)\right]+\frac{G_{m} \operatorname{Cos} \alpha}{4\left(a-K_{0} S_{c}\right)^{2}}\left[z A_{11}+2 A_{13} A_{5}\left(1-S_{c}\right)\right. \\
& \left.+2 e^{-\sqrt{a} z} A_{2} S_{c}\left(1-t K_{0}\right)-\frac{z e^{-\sqrt{a} z} A_{3} K_{0} S_{c}}{\sqrt{a}}\right]+\frac{G_{r} \operatorname{Cos} \alpha}{2 a^{2} \sqrt{\pi}} \\
& {\left[2 z a e^{\frac{-z^{2} P_{r}}{4 t}} \sqrt{t P_{r}}+\sqrt{\pi} A_{14}\left(A_{6}+A_{7} P_{r}\right)+\sqrt{\pi} A_{12}\left(a z^{2} P_{r}\right.\right.} \\
& \left.\left.-2+2 a t+2 P_{r}\right)\right]+\frac{G_{m} \operatorname{Cos} \alpha}{4 \sqrt{\pi}\left(a-K_{0} S_{c}\right)^{2}}\left[\frac{e^{-\sqrt{K_{0} S_{c}}} \sqrt{\pi} A_{9} \sqrt{S_{c}}}{2 \sqrt{K_{0}}}\right. \\
& \left(S_{c} K_{0}-a z\right)+A_{13} \sqrt{\pi} A_{10}\left(S_{c}-1\right)+e^{-\sqrt{K_{0} S_{c}}} \sqrt{\pi} A_{8}(1-a t \\
& \left.\left.-S_{c}+t K_{0} S_{c}\right)\right]
\end{aligned}
$$

The expressions for the symbols involved in the above solutions are given in the appendix.

\section{SKIN FRICTION}

The dimensionless skin friction at the plate is

$$
\left(\frac{d q}{d z}\right)_{z=0}=\tau_{x}+i \tau_{y}
$$

The numerical values of $\tau_{x}$ and $\tau_{y}$ for different parameters are given in table-1.

\section{SHERWOOD NUMBER}

The dimensionless Sherwood number at the plate is

$$
\begin{aligned}
& S_{h}=\left(\frac{\partial C}{\partial z}\right)_{z=0}=\operatorname{erfc}\left[-\sqrt{t K_{0}}\right]\left(-\frac{1}{4 \sqrt{K_{0}}} \sqrt{S_{c}}-\frac{t \sqrt{S_{c} K_{0}}}{2}\right) \\
& +\sqrt{S_{c}} \operatorname{erfc}\left[\sqrt{t K_{0}}\right]\left(\frac{1}{4 \sqrt{K_{0}}}+t \sqrt{K_{0}}\right)-\frac{e^{-t K_{0}} \sqrt{t S_{c} K_{0}}}{\sqrt{\pi K_{0}}}
\end{aligned}
$$

The numerical values of Sherwood number $S_{h}$ for different parameters are given in table-2.

\section{RESULTS AND DISCUSSION}

The present study is carried out to examine the effects of rotation and chemical reaction on the flow. The behavior of other parameters like magnetic field, Hall current and thermal buoyancy is almost similar to the earlier model studied by us [7]. The analytical results are shown graphically in figures 2 to 7 . The numerical values of skin-friction and Sherwood number are presented in Table-1and 2 respectively. Effect of rotation on flow behavior is shown by figures 2 and 3. It is observed that increase in rotation parameter leads to decrease in primary velocity throughout the boundary layer region whereas secondary velocity increases continuously near the surface of the plate. This implies that rotation tends to accelerate secondary velocity whereas it retards primary velocity in the boundary layer region. Chemical reaction effect on fluid flow behavior is shown by figures 4 and 5 . It is seen here that when chemical reaction parameter increases, primary and secondary velocities decrease throughout the boundary layer region. Further, it is observed that the concentration of the fluid near the plate decreases when chemical reaction and Schmidt number parameters are increased (figures 6 and 7).

Skin friction is given in table1. The values of $\tau_{x}$ increase with the increase in chemical reaction and rotation parameters. The values of $\tau_{y}$ increase with chemical reaction parameter and decrease with rotation parameter.

Sherwood number is given in table2. The value of $S_{h}$ decreases with the increase in the chemical reaction parameter, Schmidt number and time.

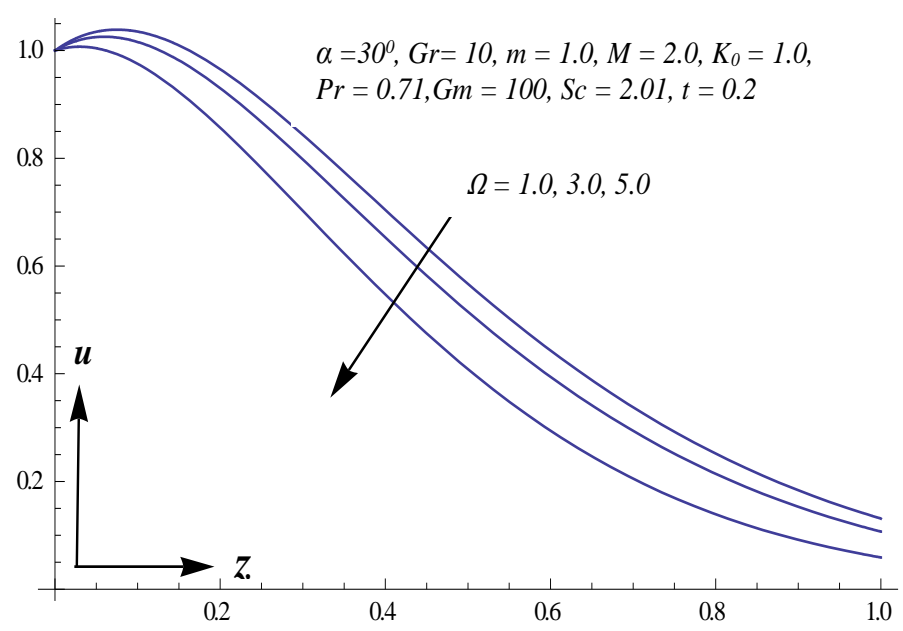

Figure 2 Velocity $u$ for different values of $\Omega$.

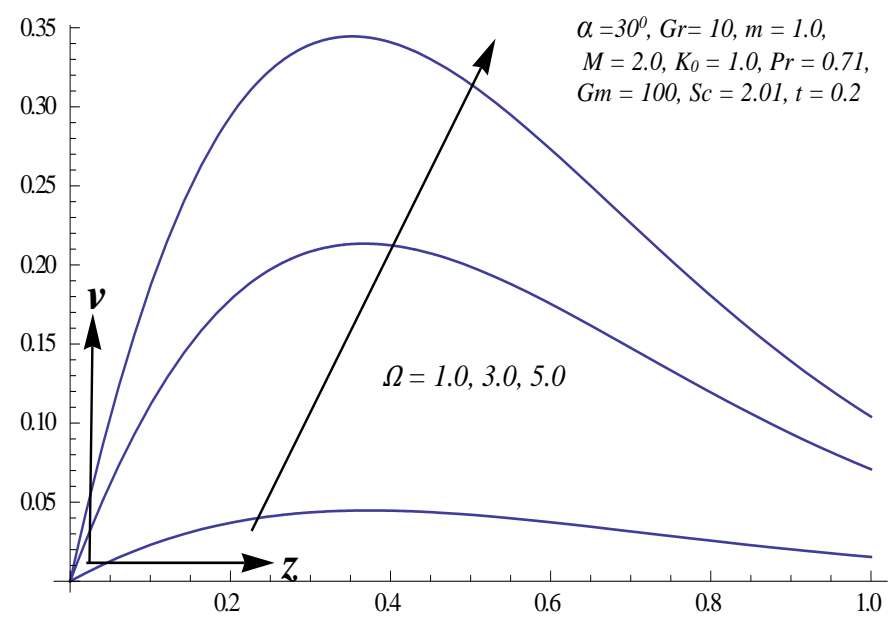

Figure 3 Velocity $v$ for different values of $\Omega$. 


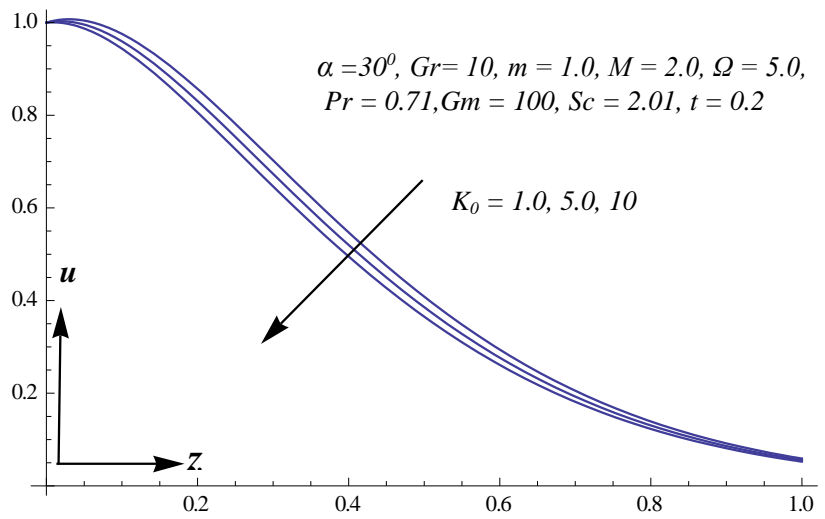

Figure 4 Velocity $u$ for different values of $K_{0}$

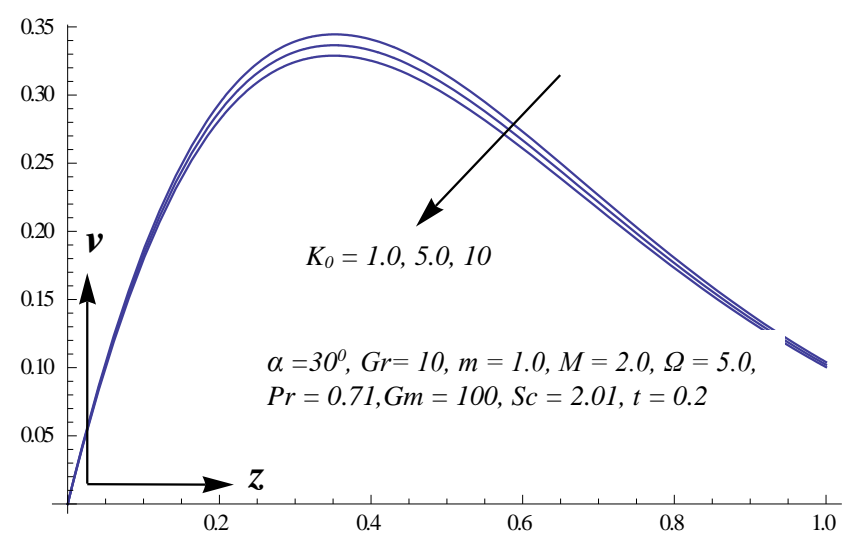

Figure 5 Velocity $v$ for different values of $K_{0}$

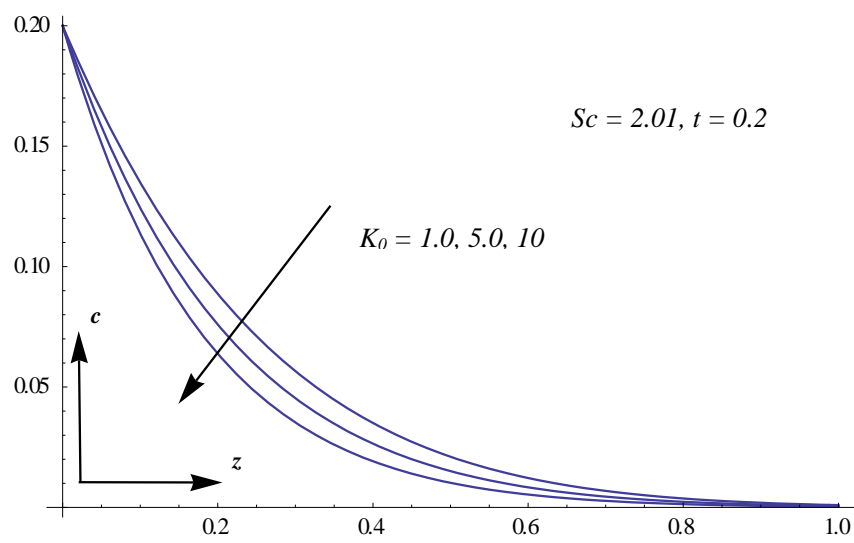

Figure 6Concentrationc for different values of $K_{0}$

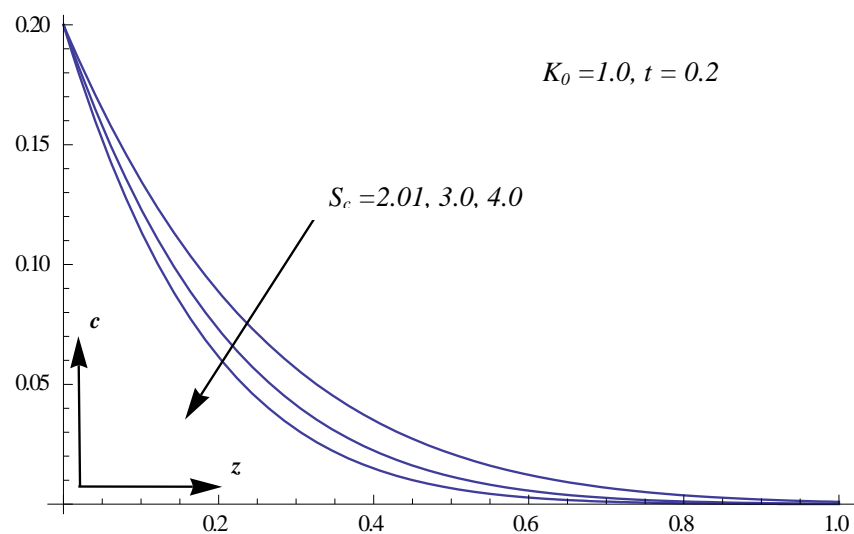

Figure 7Concentration $c$ for different values of Sc.
Table 1 Skin friction for different parameter $\left(\alpha=30^{\circ}, M=2, m=1\right)$.

\begin{tabular}{|c|c|c|c|c|c|c|c|c|}
\hline$P r$ & $S c$ & $G m$ & $G r$ & $\Omega$ & $K_{0}$ & $t$ & $\tau_{x}$ & $\tau_{y}$ \\
\hline 0.71 & 2.01 & 100 & 10 & 01 & 01 & 0.2 & - & 05.9798 \\
\hline 0.71 & 2.01 & 100 & 10 & 03 & 01 & 0.2 & - & 05.5223 \\
\hline 0.71 & 2.01 & 100 & 10 & 05 & 01 & 0.2 & - & - \\
\hline 0.71 & 2.01 & 100 & 10 & 05 & 05 & 0.2 & - & - \\
\hline 0.71 & 2.01 & 100 & 10 & 05 & 10 & 0.2 & - & - \\
\hline
\end{tabular}

Table 2 Sherwood number for different parameters.

\begin{tabular}{|c|c|c|c|}
\hline$K_{0}$ & $S c$ & $t$ & $S_{h}$ \\
\hline 01 & 2.01 & 0.2 & -0.76220 \\
\hline 05 & 2.01 & 0.2 & -0.93304 \\
\hline 10 & 2.01 & 0.2 & -1.11824 \\
\hline 01 & 3.00 & 0.2 & -0.93117 \\
\hline 01 & 4.00 & 0.2 & -1.07523 \\
\hline 01 & 2.01 & 0.3 & -0.96132 \\
\hline 01 & 2.01 & 0.4 & -1.14157 \\
\hline
\end{tabular}

\section{CONCLUSION}

In this paper a theoretical analysis has been done to study the effects of rotation and chemical reaction on unsteady MHD flow past an impulsively started inclined plate with variable wall temperature and mass diffusion in the presence of Hall current. The results obtained are in agreement with the usual flow. It has been found that the velocity in the boundary layer region decreases when chemical reaction parameter is increased. The rotation parameter retards the primary flow whereas it accelerates the secondary flow. It is also observed that chemical reaction parameter increases the drag at the plate surface, and decreases with the rotation parameter. Sherwood number decreases with increase in chemical reaction parameter. The results obtained will have applications in the research related to the solar physics dealing with the sunspot development, the structure of rotating magnetic stars, cooling of electronic components of a nuclear reactor etc.

\section{REFERENCES}

[1] A. Mahdy, Int. Commun. Heat Mass Transfer, (2010), 37 (5), 548-554.

[2] A. Sahin, A. J. Chamkha, Int. J. Industrial Mathematics,(2010), 2 (4), 245 261.

[3] D.R.V. Prasada Rao, D.V. Krishna, L. Debnath,Acta Mech, (1982), 43 (1), 49-59.

[4] R. Muthucumarswamy, P. Ganesan, Acta Mech, (2001), 147 (1-4), 45-57.

[5] S. Maripala, K. Naikoti,World Appl. Sci. J., (2015), 33 (6), 1032-1041.

[6] U. S. Rajput, N. Kanaujia, International Journal of Applied Science and Engineering, (2016), 14 (2), 115-123.

[7] U. S. Rajput, G. Kumar,Malaysian Journal of Fundamental and Applied Sciences, (2016), 12 (2), 68-76.

[8] Z. Ismail, I. Khan, A. Imran, A. Hussanan, S. Shafie, Malaysian Journal of Fundamental and Applied Sciences,(2014), 10 (1), 37-42. 


\section{APPENDIX}

$a=\frac{M(1-i m)}{1+m^{2}}+\frac{1}{K}+2 i \Omega ., A_{0}=\frac{u_{0}^{2} t}{v}$,

$A_{1}=1+A_{16}+e^{2 \sqrt{a} z}\left(1-A_{17}\right), A_{2}=-A_{1}, A_{3}=A_{16}-A_{1}$,

$A_{4}=-1+A_{22}+A_{18}\left(A_{23}-1\right), A_{5}=-1+A_{24}+A_{19}\left(A_{25}-1\right)$,

$A_{6}=-1-A_{26}+A_{18}\left(A_{27}-1\right), A_{7}=-A_{6}$,

$A_{8}=-1-A_{20}+A_{30}\left(A_{21}-1\right), A_{9}=A_{8}+2\left(A_{20}+1\right)$,

$A_{10}=-1-A_{28}+A_{19}\left(A_{29}-1\right), A_{11}=\frac{e^{-\sqrt{a z}}}{z}\left(2 A_{1}+2 a t A_{2}+\sqrt{a} A_{3}\right)$,

$A_{12}=-1+e r f\left[\frac{z \sqrt{P_{r}}}{2 \sqrt{t}}\right], A_{13}=e^{\frac{a t}{-1+S_{c}}-z \sqrt{\frac{\left(a-K_{0}\right) S_{c}}{-1+S_{c}}}-\frac{t K_{0} S_{c}}{-1+S_{c}}}$,

$A_{14}=e^{\frac{a t}{-1+P_{r}}-z \sqrt{\frac{(a) P_{r}}{-1+P_{r}}}}, A_{15}=1+A_{16}+e^{2 \sqrt{a z}} A_{17}$,

$A_{16}=\operatorname{erf}\left[\frac{2 \sqrt{a} t-z}{2 \sqrt{t}}\right], A_{17}=\operatorname{erf}\left[\frac{2 \sqrt{a} t+z}{2 \sqrt{t}}\right]$,

$A_{18}=e^{-2 z \sqrt{\frac{a P_{r}}{-1+P_{r}}}}, A_{19}=e^{-2 z \sqrt{\frac{\left(a-K_{0}\right) S_{c}}{-1+S_{c}}}}$,

$A_{21}=\operatorname{erf}\left[\sqrt{t K_{0}}+\frac{z \sqrt{S_{c}}}{2 \sqrt{t}}\right], A_{22}=\operatorname{erf}\left[\frac{z-2 t \sqrt{\frac{a P_{r}}{-1+P_{r}}}}{2 t}\right]$,

$A_{23}=\operatorname{erf}\left[\frac{z+2 t \sqrt{\frac{a P_{r}}{-1+P_{r}}}}{2 t}\right], A_{24}=\operatorname{erf}\left[\frac{z-2 t \sqrt{\frac{\left(a-K_{0}\right) S_{c}}{-1+S_{c}}}}{2 t}\right]$,

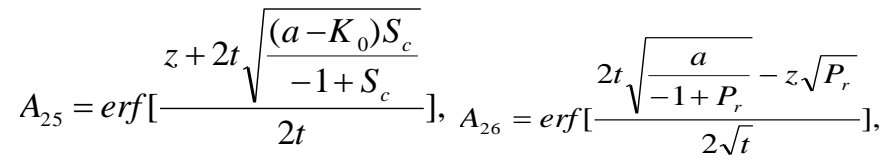

$A_{27}=\operatorname{erf}\left[\frac{2 t \sqrt{\frac{a}{-1+P_{r}}}+z \sqrt{P_{r}}}{2 \sqrt{t}}\right], A_{28}=\operatorname{erf}\left[\sqrt{t} \sqrt{\frac{\left(a-K_{0}\right)}{-1+S_{c}}}-\frac{z S_{c}}{2 \sqrt{t}}\right]$,

$A_{29}=\operatorname{erf}\left[\sqrt{t} \sqrt{\frac{\left(a-K_{0}\right)}{-1+S_{c}}}+\frac{z S_{c}}{2 \sqrt{t}}\right], A_{30}=\operatorname{erf}\left[e^{2 z \sqrt{K_{0} \sqrt{S_{c}}}}\right]$ 